\title{
Atomic Hydrogen Under Strong Soft X-Ray Pulses
}

\author{
A. R. B. de Castro \\ IFGW, UNICAMP, 13083-970, Campinas, SP, Brazil, and LNLS, CP 6192, 13083-970, Campinas, SP, Brazil
}

Received on 24 December, 2007

\begin{abstract}
We discuss theoretically the behavior of atomic Hydrogen under irradiation with strong light pulses in the soft $\mathrm{X}$-ray spectral region. The method consists in the direct numerical solution of the time dependent Schrödinger equation. We find ranges of (high) peak incident intensity $I_{0}$, where 2-photon absorption becomes more probable than 1-photon absorption. At very high intensity, the total ionization probability goes very close to 1 and then decreases as $I_{0}$ is further increased.
\end{abstract}

Keywords: Free-electron-laser; Multi-photon absorption; Hydrogen

\section{INTRODUCTION}

In a series of recent papers the effect on a Helium atom, of short intense pulses in the XUV spectral range, was discussed both theoretically and experimentally [1-5] with regard to nphoton absorption of light at $\hbar \omega_{\mathrm{ph}}=13 \mathrm{eV}$. The theoretical model contemplated the direct numerical solution of the Time Dependent Schrödinger Equation (TDSE) in a basis of finite and rather small dimensionality. In spite of the fact that the experimental results were in good agreement with the theory, the theory was questioned [6-8].

In what concerns the theory, the points of dissention seem to be (a) The use of approximate Helium electron eigenstates, which were not all orthogonal; (b) The fact that the procedure had not been tested by applying it to a well-known, previously solved problem; (c) The relevance of going beyond the Electric Dipole Moment Approximation (EDMA).

We have, therefore, applied the same procedure to the case of a Hydrogen atom, which had been discussed before using other methods. For Hydrogen, all energy eigenstates of the isolated atom are exactly known and orthogonal. All matrix elements of the interaction terms $\mathbf{p} \bullet \mathbf{A}$ and $\mathbf{A} \bullet \mathbf{A}$ for general $k_{\text {photon }}>0$ can be calculated with arbitrary numerical precision, if not as closed analytical expressions, at least as fast converging series. Exact expressions for all matrix elements are given in the Appendices. For our purpose of numerical solution of the Time Dependent Schrödinger Equation, these matrix elements were calculated to 6 significant figures and carefully checked as to convergence of the series. Then, we can also cover the EDMA case for purposes of comparison, simply by setting $k_{\text {photon }}=0$ in the general expressions.

We find fair quantitative agreement with previously published theoretical results.

\section{SCENARIO 1 FOR THE CALCULATION}

We assume the following scenario for the experiments: a photo-electron detector counts electrons resulting from ionization of the Hydrogen atoms and going into a small solid angle $\Delta \Omega$ around the $\mathbf{z}$ axis, which we choose to be parallel to the direction of the electric field $\mathbf{E}$ in the linearly polarized light beam. The radiation field is supposed to be single-mode (strictly monochromatic) with $\hbar \omega_{\mathrm{ph}}=27.21 \mathrm{eV}$ $\left(k_{\mathrm{ph}} r_{B}=0.00729, \mathbf{k}_{\mathrm{ph}}\right.$ orthogonal to $\left.\mathbf{E}=\mathrm{Ez}\right)$.

We consider only a small number of low-lying bound eigenstates and two unbound eigen-states $\left|\mathbf{k}_{1}>\right| \mathbf{k}_{2}>$ with definite $\mathbf{k}$-vector in the $\mathbf{z}$ direction; the energy eigen-values are $\mathrm{E}_{1}=$ $\hbar^{2} \mathrm{k}_{1}^{2} / 2 \mathrm{~m}_{e}$ and $\mathrm{E}_{2}=\hbar^{2} \mathrm{k}_{2}^{2} / 2 \mathrm{~m}_{e}$. We choose $\mathbf{k}_{1} \mathbf{k}_{2}$ so that both transitions (from ground state $1 \mathrm{~s}$ into $\mathbf{k}_{1}$ and from $\mathbf{k}_{1}$ into $\mathbf{k}_{2}$ ) are 1-photon resonant. An energy level diagram is shown in Fig. 1.

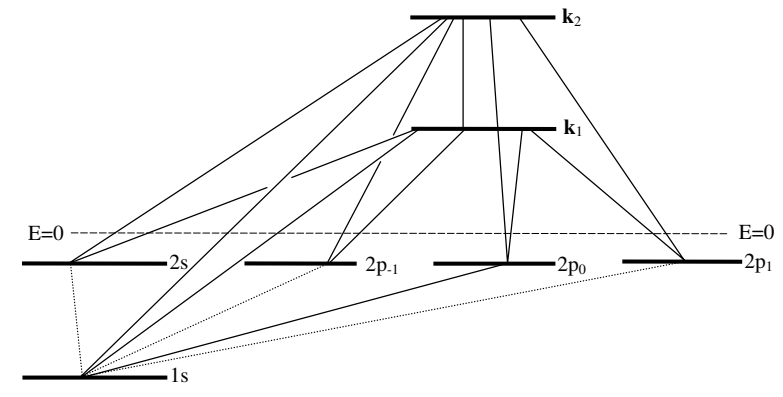

FIG. 1: Hydrogen atom energy levels (not to scale), showing all transitions possible, if exact matrix elements are considered. For the geometry considered here, however, the transitions $1 \mathrm{~s}-2 \mathrm{~s}$ and $1 \mathrm{~s}-$ $2 \mathrm{p}_{ \pm 1}$ are forbidden.

We have therefore the parameter values in Table I:

The quantized radiant field is described in terms of its energy eigenstates. Let us add here that there is no cogent reason not to use a fully quantized radiation field. In fact, it allows us to keep track very conveniently of the system states, is perfectly sound mathematically, and ... nature seems to be quantum, not classical.

We should comment on the normalization of the quasifree $(E>0)$ eigenstates of the Hydrogen atom. From the point of view of solving a system of coupled $1^{\text {st }}$ order differential equations it is much more convenient to divide up the $\mathbf{k}$ space (labels of the unbound states) into finite cells of volume $\left(\Delta^{3} \mathrm{k}\right)=\mathrm{k}^{2} \mathrm{dkd} \Omega$, define the new states $\mid \mathbf{k}>_{\text {discr }}=$ $\left(\Delta^{3} k\right)^{-1 / 2} \int_{\xi \in \Delta(k)} d^{3} \xi \mid \xi>$ and use instead of the set $\{\mid \mathbf{k}>\}$ the denumerable ortho-normal set $\left\{\mid \mathbf{k}>_{\text {discr }}\right\}$. We are interested in small values of $\left(\Delta^{3} k\right)$, describing the energy resolution and angular acceptance of the photo-electron detector.

For small $\left(\Delta^{3} k\right),\left|\mathbf{k}>_{\text {discr }} \cong\left(\Delta^{3} k\right)^{1 / 2}\right| \mathbf{k}>$; we can therefore immediately write down the matrix elements in the new basis 
TABLE I: Parameter values for the calculation in Scenario 1

\begin{tabular}{|l|l|l|l|}
\hline & Energy $(\mathrm{eV})$ & Wavevector & Other \\
\hline Photon energy $\hbar \omega_{p h}$ & 27.21 & $\mathrm{k}_{p h} \mathrm{r}_{B}=0.00729$ & $\begin{array}{l}\mathbf{k}_{p h} \bullet \mathbf{z}=0 \\
\mathbf{E} \bullet \mathbf{z}=\mathrm{E}\end{array}$ \\
\hline Ionization potential $\mathrm{IP}_{H}$ & 13.606 & & \\
\hline $\begin{array}{l}\text { Excited quasi-free state } \mathrm{E}_{1} \\
\mathrm{E}_{1}=\hbar \omega_{p h}-\mathrm{IP}_{H}\end{array}$ & 13.606 & $\mathrm{k}_{1} \mathrm{r}_{B}=1.000$ & $\mathbf{k}_{1} \bullet \mathbf{z}=\mathrm{k}_{1}$ \\
\hline $\begin{array}{l}\text { Excited quasi-free state } \mathrm{E}_{2} \\
\mathrm{E}_{2}=2 \hbar \omega_{p h}-\mathrm{IP}_{H}\end{array}$ & 40.818 & $\mathrm{k}_{2} \mathrm{r}_{B}=1.732$ & $\mathbf{k}_{2} \bullet \mathbf{z}=\mathrm{k}_{2}$ \\
\hline
\end{tabular}

as ${ }_{\text {discr }}<\mathbf{k}\left|H_{\text {int }}\right| n l m>=\left(\Delta^{3} k\right)^{1 / 2}<\mathbf{k}\left|H_{\text {int }}\right| n l m>$ and also discr $<\mathbf{k}_{2}\left|H_{\text {int }}\right| \mathbf{k}_{1}>_{\text {discr }}=\left(\Delta^{3} k_{1}\right)^{1 / 2}\left(\Delta^{3} k_{2}\right)^{1 / 2}<\mathbf{k}_{2}\left|H_{\text {int }}\right| \mathbf{k}_{1}>$.

If we consider energy resolution $\Delta E$, and angular acceptance limited to a cone of small half-angle $\theta_{m}$ about $\mathbf{k}$, then $\Delta(\mathbf{k})=\left(\pi \theta_{m}^{2}\right) k^{3}(1 / 2 \Delta E / E)$. Take $\Delta \mathrm{E} / \mathrm{E}=0.1, \theta_{m}=0.174 \mathrm{rad}$ [5]. Then $\left(\Delta^{3} \mathrm{k}_{1}\right)=43.2 \times 10^{-4} \mathrm{r}_{B}^{-3}$ and $\left(\Delta^{3} \mathrm{k}_{2}\right)=224.5 \times 10^{-4}$ $\mathrm{r}_{B}^{-3}$.

Details of the calculation have been given elsewhere [1]; in short, we go over to the interaction picture [9] and solve a linear system of $1^{\text {st }}$ order differential equations numerically, using the Hairer and Wanner [10] code. Let $\mathrm{H}^{\prime}$ be the interaction term in the Hamiltonian. In dimensionless form (atomic units), the system of equations is

$$
\begin{aligned}
i \partial / \partial t C_{n}(t) & =\sum_{m} \exp \left(i \omega_{n m} t\right)<n\left|H^{\prime \prime}\right| m>C_{m}(t) \\
\omega_{n m} & =\left(E_{n}--E_{m}\right) /(2 \mathrm{Rydberg}) \\
H^{\prime \prime} & =H^{\prime} /(2 \text { Rydberg })
\end{aligned}
$$

Here $H^{\prime \prime}$ has two contributions. The first connects field states differing by 1 photon, while the second connects field states differing by 2 photons:

$$
\begin{aligned}
H_{p . A}^{\prime \prime} & =(1 / 2 m)(e / c)<\text { fin }|2 \mathbf{A} \bullet \mathbf{p}| \text { ini }>/(2 \text { Rydberg })= \\
& =i\left(1.76610^{-12} \operatorname{erg}^{-1 / 2} \sec ^{1 / 2} \mathrm{~cm} \sqrt{ } \mathrm{I}\right)<\text { fin }\left|\exp \left(i \mathbf{k}_{p h} \bullet \mathbf{r}\right) \partial / \partial z\right| i n i> \\
H_{A . A}^{\prime \prime} & =(1 / 2 m)(e / c)^{2}<\text { fin }|\mathbf{A} \bullet \mathbf{A}| \text { ini }>/(2 \text { Rydberg })= \\
& =\left(1.56110^{-24} \mathrm{erg}^{-1} \operatorname{seccm}^{2} \mathrm{I}\right)<\text { fin }\left|\exp \left(2 i \mathbf{k}_{p h} \bullet \mathbf{r}\right)\right| \text { ini }>
\end{aligned}
$$

where $I$ is the intensity (peak power density/unit area) in units of $\mathrm{erg} \mathrm{sec}^{-1} \mathrm{~cm}^{-2}$.

We assumed a smooth bell-shaped envelope for the laser pulse intensity, with peak power density $I_{0}$ :

$$
\begin{aligned}
& \mathrm{I}(t)=\mathrm{I}_{0} \sin ^{2}[3 \pi t / 2 T] \quad \text { if } \quad 0<t<T / 3 \\
& =\mathrm{I}_{0} \quad \text { if } T / 3<t<2 T / 3 \\
& =\mathrm{I}_{0} \sin ^{2}[3 \pi(T-t) / 2 T] \text { if } 2 T / 3<t<T
\end{aligned}
$$

The index $\mathrm{n}$ in $\mathrm{C}_{n}(\mathrm{t})$ runs over a double set of 7 orbital states ( $\left.1 \mathrm{~s}, 2 \mathrm{~s}, 2 \mathrm{p}_{1}, 2 \mathrm{p}_{0}, 2 \mathrm{p}_{-1}, \mathbf{k}_{1}, \mathbf{k}_{2}\right)$ and 4 photon states $\mathrm{N}-\mathrm{j}$, $\mathrm{j}=0,1,2,3$ where $\mathrm{N}$ is the number of photons in the (singlemode) field, and $\mathrm{j}$ is the number of photons absorbed by the Hydrogen atom. So, there is a total of 21 complex functions $\mathrm{C}_{n}(\mathrm{t})$ to be determined. The system is solved under the initial condition that the atom is in the ground state.

We have extensively checked the correctness of the numerical solutions in the context of quantum mechanics: it was, for instance verified that the system state norm is kept invariant to better than 1 part in $10^{6}$, that the occupation probabilities do not depend on the phase of the initial condition, etc.
We have more recently performed additional tests. For instance, we can disable all transitions except one. In this way, we can simulate a two-level system, under resonant or nonresonant conditions, for absorption of one or two photons, under excitation with arbitrary time envelopes.

For either 1-photon absorption or 2-photon absorption, in the weak excitation limit, with fixed square or bell-shaped laser pulse envelope, under resonant conditions, we find that the occupation probability of the excited state depends on intensity linearly (1-photon absorption) or quadratically (2photon absorption), as expected.

For either 1-photon absorption or 2-photon absorption, in the strong excitation limit, with long square pulse envelope, under resonant or non-resonant conditions, the occupation probability of the excited state, given by the numerical solution of the TDSE, is found to depend on time, on the "detuning" $\omega_{n m}$ and on field intensity just as predicted by the Rabi equations [9].

We want to compare our results with the calculation by Nurhuda and Faisal [11], where the Electric Dipole Moment Approximation (EDMA) is used.

Table II gives the numerical values of the matrix elements 
$<$ fin $\left|\exp \left(i \mathbf{k}_{\mathrm{ph}} \bullet \mathbf{r}\right) \partial / \partial z\right|$ ini $>$ calculated according to the general formulae in the Appendices (and setting $k_{\mathrm{ph}}=0$ ). In the EDMA, $<$ fin $\left|\exp \left(2 i \mathbf{k}_{\mathrm{ph}} \bullet \mathbf{r}\right)\right| i n i>=0$.

TABLE II: Matrix elements $\Delta$ (EDMA) for comparison with ref [11].

\begin{tabular}{|l|l|}
\hline Transition & $\begin{array}{l}\text { Normalized dimensionless matrix el- } \\
\text { ement } \mathrm{M}\end{array}$ \\
\hline $2 \mathrm{~s} \leftarrow 1 \mathrm{~s}$ & 0 \\
\hline $2 \mathrm{p}_{ \pm 1} \leftarrow 1 \mathrm{~s}$ & 0 \\
\hline $2 \mathrm{p}_{0} \leftarrow 1 \mathrm{~s}$ & -0.2793 \\
\hline $\mathrm{k}_{1} \mathbf{z} \leftarrow 1 \mathrm{~s}$ & $-0.009346 \mathrm{i}$ \\
\hline $\mathrm{k}_{1} \mathbf{z} \leftarrow 2 \mathrm{~s}$ & $-0.004445 \mathrm{i}$ \\
\hline $\mathrm{k}_{1} \mathbf{z} \leftarrow 2 \mathrm{p}_{ \pm 1}$ & 0 \\
\hline $\mathrm{k}_{1} \mathbf{z} \leftarrow 2 \mathrm{p}_{0}$ & 0.0005552 \\
\hline $\mathrm{k}_{2} \mathrm{z} \leftarrow 1 \mathrm{~s}$ & -0.004746 \\
\hline $\mathrm{k}_{2} \mathrm{z} \leftarrow 2 \mathrm{~s}$ & $0.001921 \mathrm{i}$ \\
\hline $\mathrm{k}_{2} \mathbf{z} \leftarrow 2 \mathrm{p}_{ \pm 1}$ & 0 \\
\hline $\mathrm{k}_{2} \mathrm{z} \leftarrow 2 \mathrm{p}_{0}$ & 0.00007007 \\
\hline $\mathrm{k}_{2} \mathrm{z} \leftarrow \mathrm{k}_{1} \mathrm{z}$ & $-0.00007503 \mathrm{i}$ \\
\hline
\end{tabular}

The quantity needed for the numerical solution is $\gamma=\mathrm{C}_{p . A}$ $\mathrm{M} \sqrt{ } \mathrm{I}_{0}$, where $\mathrm{I}_{0}$ is the maximum intensity in units of $10^{12}$ $\mathrm{W} / \mathrm{cm}^{2}$ and $\mathrm{C}_{p . A}=0.005584 \mathrm{i}$.

We used $\mathrm{T}=93$ atomic units of time and a field intensity range $1<\mathrm{I}_{0}<120000\left(\mathrm{x} 10^{12} \mathrm{~W} / \mathrm{cm}^{2}\right)$, which spans the region up to and beyond the intensity considered in [11]. We focus on the occupation probabilities of the quasi-free orbital states $\mathbf{k}_{1} \mathbf{k}_{2}$ for comparison with the results in Fig. 1 of ref [11].

There are small differences in the normalization of the quasi-free states used here and in ref [6]; we think these are of minor significance, but are expected to change somewhat the numerical values.

Our discussion is centered on the radiant field intensitydependence of the occupation probabilities after the excitation pulse is turned OFF, and on the time-dependence of the projections while the laser pulse is ON. The discussion of Nurhuda and Faisal [11] is centered on the electron-energy dependence of the ionization rate after the excitation pulse is finished, but at the single fixed radiation field intensity of $3.5 \times 10^{16} \mathrm{~W} / \mathrm{cm}^{2}$.

Figure 2 shows the dependence on field intensity of the occupation probability of the quasi-free states $\mathbf{k}_{1}$ and $\mathbf{k}_{2}$ immediately after the excitation pulse is finished.

At lower field intensity, the probability grows linearly with intensity, as expected for a 1-photon absorption process. The matrix elements for transitions from $1 \mathrm{~s}$ into $\mathbf{k}_{1}$ or $\mathbf{k}_{2}$ have almost the same values, but the transition into $\mathbf{k}_{1}$ is resonant, while the transition into $\mathbf{k}_{2}$ is not. The large detuning causes the projection of the system state on $\mathbf{k}_{2}$ to oscillate during the excitation pulse, and the net transfer of population into $\mathbf{k}_{2}$ is very small.

At large field intensity, we witness strong non-linear and non-monotonic behavior of the probabilities $\mathrm{P}\left(\mathbf{k}_{1}\right) \mathrm{P}\left(\mathbf{k}_{2}\right)$, as found before [1]. There are narrow ranges of field intensities

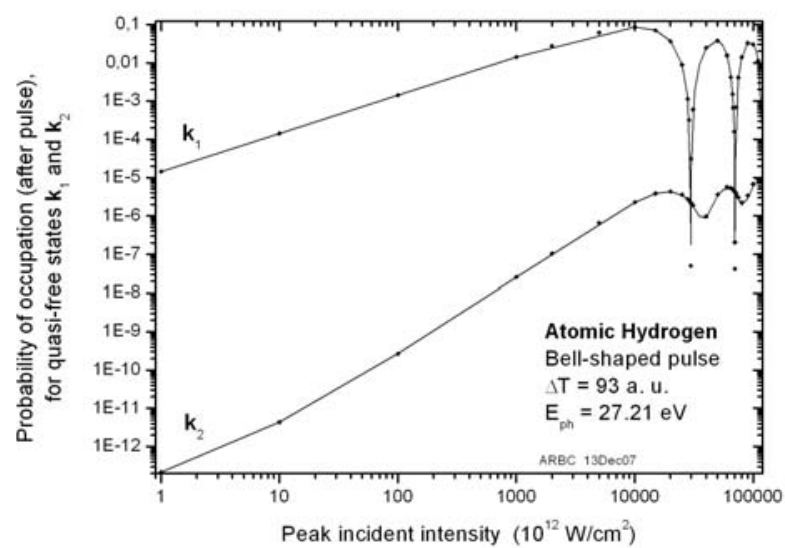

FIG. 2: Probability of occupation for the atomic Hydrogen quasi-free states $\mathbf{k}_{1}$ and $\mathbf{k}_{2}$, immediately after completion of the light pulse. The horizontal axis covers the range $10^{12}$ up to $10^{17} \mathrm{~W} / \mathrm{cm}^{2}$. The light pulse is bell-shaped, with total duration of 93 atomic units of time and photon energy $E_{\mathrm{ph}}=27.21 \mathrm{eV}$. The full lines are the result of a calculation in the Electric Dipole Moment Approximation. The full diamonds are the result of a calculation with exact matrix elements for both terms $\mathbf{p} \bullet \mathbf{A}$ and $\mathbf{A} \bullet \mathbf{A}$ of the interaction.

(for instance, 3.0 to $4.0 \times 10^{16} \mathrm{~W} / \mathrm{cm}^{2}$ ) where the $\mathbf{k}_{1}$ population $\mathrm{P}\left(\mathbf{k}_{1}\right)$ changes very rapidly, and goes successively thru values much larger and much smaller than the $\mathbf{k}_{2}$ population $\mathrm{P}\left(\mathbf{k}_{2}\right)$. Nurhuda and Faisal find $\mathrm{P}\left(\mathbf{k}_{1}\right) / \mathrm{P}\left(\mathbf{k}_{2}\right) \sim 10$ at $3.5 \times 10^{16}$ $\mathrm{W} / \mathrm{cm}^{2}$ ), while in the present calculation, this happens at $3.0 \times 10^{16} \mathrm{~W} / \mathrm{cm}^{2}$. We attribute the difference to distinct criteria for normalization of the quasi-free states; while we normalized to the acceptance $\Delta^{3} k$ of a typical electron analyzer placed along the direction of the radiant electric field vector $\boldsymbol{E}$, ref [6] does not specify the normalization of the states or the experimental arrangement.

Figure 3 shows the time development of the system state projections on the state $\mathbf{k}_{1}$. Let us observe that while the radiant field is ON, we cannot speak of "electron state" because the electron is tightly coupled to the radiation field. However, the system state can still be written as a superposition of eigenstates from the isolated atom and the free radiation field, and we can follow the evolution of the projections. At lower field intensities, the projection on $\mathbf{k}_{1}$ grows smooth and monotonically, as expected from elementary perturbation theory. However, a complicated non-monotonic behavior is evident at high field intensity.

Regarding this complex behavior, let us observe that all two-level systems under harmonic steady-state excitation are exactly described by the Rabi equations [9]. These equations predict non-linear non-monotonic dependence of the populations on field intensity. Here, on the other hand, we are discussing a n-level $(n>2)$ system excited by very short bellshaped pulses, not a steady-state excitation. Furthermore, here the pulse length and the Rabi period for the transitions are comparable, and we expect a complicated interplay of these various effects.

We have also tried to ascertain the importance of the corrections to the EDMA. The full diamonds in Fig. 2 are the 


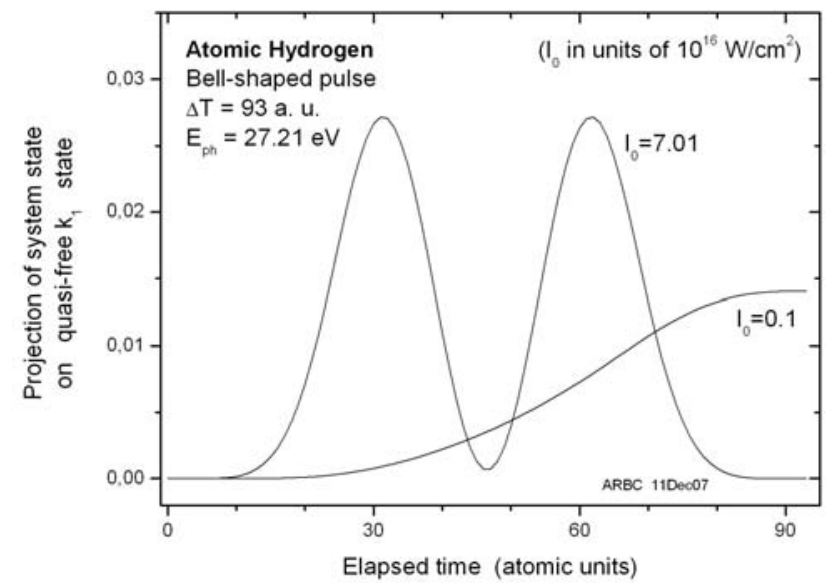

FIG. 3: Time-dependence of the system-state projection on the electron quasi-free state $\mathbf{k}_{1}$, at low incident intensity and at high incident intensity. In the former case we see a smooth monotonic increase of the projection, while in the latter case, a complicated non-monotonic evolution. At low intensity, the Rabi period is much longer than the pulse length. At high intensity, the pulse length and Rabi period have values in the same order-of-magnitude.

result of the exact calculation for the matrix elements of $\mathbf{p} \bullet \mathbf{A}$ and $\mathbf{A} \bullet \mathbf{A}$, setting $\mathrm{k}_{\mathrm{ph}}=0.00729$. It is clear from Fig. 2 that in Hydrogen these corrections are significant only for the highest intensities $\left(\mathrm{I}_{0} \sim 10^{16} \mathrm{~W} / \mathrm{cm}^{2}\right)$ and affect mostly the probability of occupation of the quasi-free state $\mathbf{k}_{2}$, which requires 2-photon absorption in order to be populated.

Let us also observe that the deep minima shown in Fig. 2 are not believed to be experimentally observable, in so far as the experimental apparatus will collect not only photo-electrons going into the sharply defined $\mathbf{k}_{1}$ (or $\mathbf{k}_{2}$ ) state, but also going into neighboring quasi-free states, within some energy acceptance. In the next section we address this question.

It is seen that our procedure, when applied to atomic Hydrogen, under the conditions considered by Nurhuda and Faisal [11], reproduces their result regarding the ratio of probabilities for 1-photon and 2-photon absorption, except for a small shift $(15 \%)$ in the value of the radiant field intensity. On the other hand, our calculation shows that the probabilities are changing very rapidly and by several orders of magnitude in this region of field intensities. A more detailed comparison would need more complete information about the normalization of the quasi-free electron states in [11], which has not been given.

\section{SCENARIO 2 FOR THE CALCULATION}

Here we would like to compare our results with those of Bauer Plucinski Piraux Potvliege Gajda and Krzywinski [12]. They also used EDMA, with different pulse profile and intensity range.

We have here the parameter values given in Table III:

The normalization constants are now $\left(\Delta^{3} k_{1}\right)^{1 / 2}=0.1375$ and $\left(\Delta^{3} k_{2}\right)^{1 / 2}=0.2629$.
The pulse length here is $\mathrm{T}=103$ atomic units while the pulse profile is

$$
\mathrm{I}(t)=\mathrm{I}_{0} \sin ^{2}(\pi t / T) \quad \text { for } \quad 0<t<T,
$$

The intensity independent dimensionless matrix elements $\mathrm{M}$ are given in Table IV below.

Tables II and IV show that the matrix elements $\mathrm{M}$ change little on going from $\hbar \omega=27.21 \mathrm{eV}$ to $\hbar \omega=50 \mathrm{eV}$. However, for given $\mathrm{M}$ and $\hbar \omega$, the transition rates are strongly affected by the resonant or non-resonant character of the process. So, we simulate the effect of adjoining quasi-free levels by repeating the calculation with the same matrix elements $M$ but introducing energy displacements $\delta \mathrm{E}$ in the quantities $\omega_{n m}$. Then we average over the group of adjoining quasi-free states. For each value of $\mathrm{I}_{0}$, we used groups of 5 quasi-free states, with $\left(\delta E_{1}, \delta E_{2}\right)=(0,0),(\varepsilon, 0),(-\varepsilon, 0),(0, \varepsilon),(0,-\varepsilon)$ and $\varepsilon=2 \mathrm{eV}$, a value which is consistent with the normalization of the quasifree states.

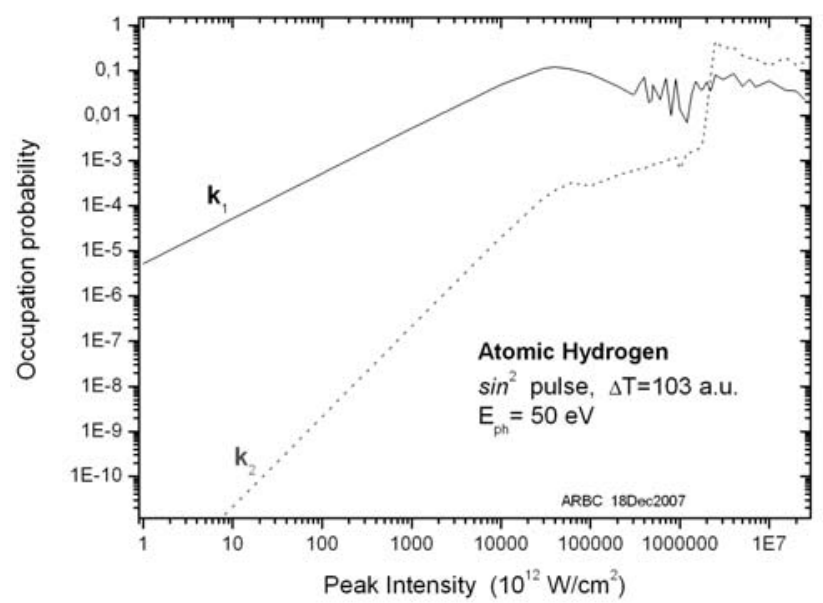

FIG. 4: Probability of occupation for a group of 5 atomic Hydrogen quasi-free states $\mathbf{k}_{1}$ and $\mathbf{k}_{2}$, separated by $\delta \mathrm{E}=0, \pm 2 \mathrm{eV}$, immediately after completion of the light pulse. The horizontal axis covers the range $10^{12}$ up to $2.510^{19} \mathrm{~W} / \mathrm{cm}^{2}$.

The light pulse is bell-shaped $\left(\sin ^{2}\right)$, with total duration of 103 atomic units of time and photon energy $E_{p h}=50 \mathrm{eV}$. The full lines are the result of a calculation in the Electric Dipole Moment Approximation. Notice the sudden increase in the population of $\mathbf{k}_{2}$ at about $2.510^{18} \mathrm{~W} / \mathrm{cm}^{2}$, and the slopes 12 in the log-log plot, characteristic of 1-photon and 2-photon absorption at low intensity.

Figure 4 shows our results for the average occupation of each group (centered on $\mathbf{k}_{1}$ and $\mathbf{k}_{2}$, respectively). In our model with a finite number of accessible orbital states, and for the experimental scenario described above, the total ionization probability per atom per incident pulse is the sum of the probabilities for going into the groups $\mathbf{k}_{1}$ and $\mathbf{k}_{2}$.

The figure shows that at low intensities, the population in group $\mathbf{k}_{1}$ (transition assisted by 1-photon absorption) is orders of magnitude larger than in group $\mathbf{k}_{2}$ (transition assisted by 2-photon absorption), as expected from perturbation theory. The slopes in the log-log plot are as expected, namely, exactly 1 and 2. 
TABLE III: Parameter values for the calculation in Scenario 2.

\begin{tabular}{|l|l|l|l|}
\hline & Energy $(\mathrm{eV})$ & Wavevector & Other \\
\hline Photon energy $\hbar \omega_{p h}$ & 50.0 & $\begin{array}{l}\mathrm{k}_{p h} \mathrm{r}_{B}=0 \\
\text { (EDMA) }\end{array}$ & $\begin{array}{l}\mathbf{k}_{p h} \bullet \mathbf{z}=0 \\
\mathbf{E} \bullet \mathbf{z}=\mathrm{E}\end{array}$ \\
\hline Ionization potential $\mathrm{IP}_{H}$ & 13.606 & & \\
\hline $\begin{array}{l}\text { Excited quasi-free state } \mathrm{E}_{1} \\
\mathrm{E}_{1}=\hbar \omega_{\mathrm{ph}}-\mathrm{IP}_{H}\end{array}$ & 36.394 & $\mathrm{k}_{1} \mathrm{r}_{B}=1.636$ & $\mathbf{k}_{1} \bullet \mathbf{z}=\mathrm{k}_{1}$ \\
\hline $\begin{array}{l}\text { Excited quasi-free state } \mathrm{E}_{2} \\
\mathrm{E}_{2}=2 \hbar \omega_{\mathrm{ph}}-\mathrm{IP}_{H}\end{array}$ & 86.394 & $\mathrm{k}_{2} \mathrm{r}_{B}=2.520$ & $\mathbf{k}_{2} \bullet \mathbf{z}=\mathrm{k}_{2}$ \\
\hline
\end{tabular}

TABLE IV: Matrix elements $\Delta$ (EDMA) for comparison with ref [12].

\begin{tabular}{|l|l|}
\hline Transition & $\begin{array}{l}\text { Normalized dimensionless matrix el- } \\
\text { ement } \mathrm{M}\end{array}$ \\
\hline $2 \mathrm{~s} \leftarrow 1 \mathrm{~s}$ & 0 \\
\hline $2 \mathrm{p}_{ \pm 1} \leftarrow 1 \mathrm{~s}$ & 0 \\
\hline $2 \mathrm{p}_{0} \leftarrow 1 \mathrm{~s}$ & -0.2793 \\
\hline $\mathrm{k}_{1} \mathbf{z} \leftarrow 1 \mathrm{~s}$ & $-0.009864 \mathrm{i}$ \\
\hline $\mathrm{k}_{1} \mathbf{z} \leftarrow 2 \mathrm{~s}$ & $-0.004045 \mathrm{i}$ \\
\hline $\mathrm{k}_{1} \mathbf{z} \leftarrow 2 \mathrm{p}_{ \pm 1}$ & 0 \\
\hline $\mathrm{k}_{1} \mathbf{z} \leftarrow 2 \mathrm{p}_{0}$ & 0.0001699 \\
\hline $\mathrm{k}_{2} \mathbf{z} \leftarrow 1 \mathrm{~s}$ & -0.007276 \\
\hline $\mathrm{k}_{2} \mathbf{z} \leftarrow 2 \mathrm{~s}$ & $0.002761 \mathrm{i}$ \\
\hline $\mathrm{k}_{2} \mathrm{z} \leftarrow 2 \mathrm{p}_{ \pm 1}$ & 0 \\
\hline $\mathrm{k}_{2} \mathrm{z} \leftarrow 2 \mathrm{p}_{0}$ & 0.00003754 \\
\hline $\mathrm{k}_{2} \mathbf{z} \leftarrow \mathrm{k}_{1} \mathbf{z}$ & $-0.001736 \mathrm{i}$ \\
\hline
\end{tabular}

The figure also shows that at high intensities (above about $10^{16} \mathrm{~W} / \mathrm{cm}^{2}$ ) the behavior is non-monotonic. At about $10^{18}$ $\mathrm{W} / \mathrm{cm}^{2}$ the population of the $\mathbf{k}_{2}$ group shoots suddenly up and exceeds the population of the $\mathbf{k}_{1}$ group by roughly a factor of 10. The two populations then go down slowly as the incident field intensity is further increased.

It is not so easy to compare these results with ref [12] because it is not clear what the authors mean by "ionization rate [a.u.]" in their figure 3, where the maximum value is $\sim 0.07$ at $\mathrm{I}_{0}=4 \times 10^{17} \mathrm{~W} / \mathrm{cm}^{2}$, or "total ionization yield" in their figure 4 , where a very broad maximum value $\sim 0.95$ is found at $2 \times 10^{17} \mathrm{~W} / \mathrm{cm}^{2}<\mathrm{I}_{0}<2 \times 10^{18} \mathrm{~W} / \mathrm{cm}^{2}$. Our data gives a maximum ionization probability per atom per pulse $\sim 0.54$ at $\mathrm{I}_{0}=$ $2.5 \times 10^{18} \mathrm{~W} / \mathrm{cm}^{2}$.

Figures 5 and 6 in ref [12] give also photo-electron spectra at incident intensities $\mathrm{I}_{0}=2.5 \times 10^{18} \mathrm{~W} / \mathrm{cm}^{2}$ and $\mathrm{I}_{0}=$ $2.5 \times 10^{19} \mathrm{~W} / \mathrm{cm}^{2}$, both within the range of the present calculation. There is clear disagreement between the results at $\mathrm{I}_{0}=$ $2.5 \times 10^{19} \mathrm{~W} / \mathrm{cm}^{2}$, since our calculation (see our figure 4) predicts a 2-photon peak larger than the 1-photon peak, while ref [12] predicts a 1-photon peak larger than the 2-photon peak. Regarding the spectra at $\mathrm{I}_{0}=2.5 \times 10^{18} \mathrm{~W} / \mathrm{cm}^{2}$, the comparison is inconclusive, because our occupation probabilities are changing very fast at precisely this field intensity. The position (along the intensity axis) of the cross-over is expected to depend on the normalization of our quasi-free states, as explained before.

There are other differences in the calculations, which may or may not have a bearing on these comparisons. We used exact Coulomb wave-functions with definite $\mathbf{k}$ for the quasifree states, which are an infinite sum of spherical waves. A large number of such waves was needed to reach convergence with 6 significant figures. The authors of ref [12], on the other hand, used the semi-classical approximation to set up the TDSE and solved it on a basis of Sturmian functions, which are not eigen-functions of the isolated hydrogen atom. Also, we explicitly address a situation where the photo-electron detector, although placed in the preferential direction for photoionization, collects electrons only on a restricted solid angle.

\section{DISCUSSION AND CONCLUSION}

The non-monotonic dependence on incident light intensity, of occupation probabilities for quasi-free electron states involving absorption of $1,2, \ldots$ photons, found in the $\mathrm{He}-$ lium calculation [1] is evident also in the present calculation. Here, however, only exact eigen-states were used; the nonmonotonic behavior just mentioned cannot therefore be associated with faulty approximate eigen-states, as has been suggested in the case of Helium [6,7].

For Hydrogen, and for the radiant field intensity range considered in ref [11], the EDMA seems quite adequate. This shows also that the non-monotonic behavior of the probabilities for 1-photon and 2-photon absorption (at least in Hydrogen) do not depend exclusively on the exact forms of the $\mathbf{p} \bullet \mathbf{A}$ and $\mathbf{A} \bullet \mathbf{A}$ interaction terms. Upon consideration of the detailed time-dependence of the system-state projections it is clear that, for sufficiently short and powerful laser pulses, many quasi-free states will be populated whether or not the transition is resonant. Physically, this is completely consistent with the fact that a sufficiently short pulse cannot be strictly monochromatic.

Regarding the calculation of the matrix elements, where misleading symmetry arguments are often invoked to claim they should vanish, let us observe the following:

1) The matrix element of $\mathbf{A} \bullet \mathbf{A}$ is non-vanishing in general, although it is zero when $k_{\text {photon }}=0$ and the initial and final states are orthogonal. Therefore, even for small $k_{\text {photon}}$, it may be important to keep the leading non-vanishing contribution of $\mathbf{A} \bullet \mathbf{A}$, as we did in [1], specially if one is contemplating 
very high peak power density applications. This is in sharp contrast to the opinion of our critics [6-8].

2) If the matrix element of p.A has a finite value at $\mathrm{k}_{\text {photon }}=0$, the next correction is, for initial and final states of usual interest and current FEL wavelengths, comparatively very small. Here, it seems quite safe to use the EDMA $\left(k_{\text {photon }}=0\right)$ without further ado, as most text-books teach, and as our critics [6-8] hold to be the correct way to deal with matter-light calculations in a regime similar to the one we contemplated in [1].

Regarding comparison with previous theoretical results in the literature, we find quantitative agreement within $15 \%$ for the relative intensities of photo-electron peaks at intensities close to $3.5 \times 10^{16} \mathrm{~W} / \mathrm{cm}^{2}$, resulting from 1 and 2 photon absorption as calculated by Nurhuda and Faisal [11]. We find qualitative agreement with ref [12] in so far as both calculations predict almost complete (95\% in ref [12], 54\% in our calculation) ionization at intensities close to $2.5 \times 10^{18} \mathrm{~W} / \mathrm{cm}^{2}$, and a "stabilization" effect (reduction in the ionization yield as the intensity is even further increased). We disagree on the relative intensities of the photo-electron peaks at $2.5 \times 10^{19}$ $\mathrm{W} / \mathrm{cm}^{2}$. As far as the author knows, there are not yet experimental results to check such calculations.

\section{Acknowledgements}

ARBC acknowledges financial help from FAPESP for visits to TU Berlin and HASYLAB am DESY, where part of these calculations was done. He also acknowledges many fruitful discussions with Thomas Möller (TU Berlin) which were essential to weed out programming mistakes and clarify the physical meaning of the results. He also thanks the two institutions for making his stay in Germany very pleasant indeed.

\section{Appendix 1: definitions and useful formulae}

The exact normalized energy eigenstates for Hydrogen are (using spherical coordinates $r \theta \varphi$, with $\mathrm{r}$ given in units of $r_{B}$ and $k$ in units of $r_{B}^{-1} ;{ }_{1} F_{1}$ are confluent hypergeometric functions)

(eq. (1.1); bound states with definite $l m$ )

$$
\begin{gathered}
\mid n l m>=f_{n l}(r) Y_{l m}(\theta \varphi) \\
f_{n l}(r)=N_{n l}(2 r / n)^{l} \exp (-r / n){ }_{1} F_{1}(1+l-n, 2+2 l, 2 r / n) \\
N_{n l}=2\left[n^{2}(2 l+1) !\right]^{-1}[(n+l) ! /(n-l-1) !]^{1 / 2}
\end{gathered}
$$

(eq. (1.2); unbound Coulomb states with definite $l m$ )

$$
\begin{aligned}
\mid k, l m>= & C(k, l)(2 i k r){ }^{l} \exp (-i k r) \\
& { }_{1} F_{1}(1+l+i / k, 2+2 l, 2 i k r) Y_{l m}(\theta \varphi) \\
C(k, 0)= & (2 / \sqrt{ } k)[1-\exp (-2 \pi / k)]^{-1 / 2} \\
C(k, l)= & (2 / \sqrt{ } k)[1-\exp (-2 \pi / k)]^{-1 / 2} \\
& {[l ! /(1+2 l) !] \prod_{s=1, l}\left[1+(k s)^{-2}\right]^{1 / 2} \quad(l>0) }
\end{aligned}
$$

The unbound Coulomb states with definite $l m$ are formally obtained from the bound states replacing $1 / n$ with $i k$ and $N_{n l}$ with $C(k, l)$.

(eq. (1.3); unbound Coulomb states with definite $\mathbf{k}$ )

$$
\left|\mathbf{k}>=\sum_{l m} Y_{l m}\left(\mathbf{e}_{k}\right) *\right| k, \operatorname{lm}>
$$

The energy eigenvalues are $E_{n}=-E_{0} / n^{2}$, with $E_{0}=e^{2} / r_{B}$ for the bound states and $E(k)=(\nabla k)^{2} / 2 m_{e}$ for the unbound states. They are degenerate with respect to the orbital angular momentum quantum numbers $l m$.

The radial function of the unbound states, $\left\{(2 k r)^{l} \exp (-i k r)_{1} F_{1}(1+l+i / k, 2+2 l, 2 i k r)\right\}$ (with no $i$ factor in $\left.(2 k r)^{l}\right)$, has been shown to be real [13].

The following developments will be useful:

$$
\exp (i \mathbf{k} . \mathbf{r})=4 \pi \sum_{l m} i^{l} j_{l}(k r) Y_{l m}(\theta \varphi) Y_{l m}\left(\mathbf{e}_{\mathbf{k}}\right) *
$$




$$
\begin{gathered}
j_{S}(u)=\left[u^{S} /(2 S+1) ! !\right] \exp (-i u)_{1} F_{1}(1+S, 2+2 S, 2 i u) \\
j_{S}(u)=\sum_{q=0}^{\infty} b_{q}^{(S)} u^{2 q+S}
\end{gathered}
$$

with

$$
\begin{gathered}
b_{q}^{(S)}=2^{S}(-1)^{q}(q+S) ! /[(1+2 q+2 S) ! q !] \\
j_{S}(u)=\sum_{q=0}^{S+1} u^{-q}\left[A_{q}^{(S)} \exp (i u)+B_{q}^{(S)} \exp (-i u)\right]
\end{gathered}
$$

The $A_{q}^{(S)} B_{q}^{(S)}$ follow from the recursion relations of the spherical Bessel functions.

We adopt the following convention for the phase of the spherical harmonic functions $Y_{l m}$ :

$$
\begin{gathered}
Y_{l m}(\theta \varphi)=W_{l m} P_{l|m|}(\cos \theta) \exp (i m \varphi) \\
W_{l m}=(-1)^{(m+|m|) / 2}[(2 l+1) / 4 \pi]^{1 / 2}[(l-|m|) ! /(l+|m|) !]^{1 / 2}
\end{gathered}
$$

The following identities for hypergeometric functions [15] were extensively used in the calculation of matrix elements:

$$
\begin{gathered}
{ }_{1} F_{1}(-n, c, z)=\sum_{v=0}\left[(-n)_{v} /\left(c_{v} v !\right)\right] z^{v} \quad \text { if } n \geq 0 \text { is an integer } \\
{ }_{1} F_{1}(a, c, z)=\Gamma(c) \Gamma(a)^{-1} \Gamma(c-a)^{-1} \quad \int_{0}^{1} d t e^{z t} t^{a-1}(1-t)^{c-a-1} \quad \text { if } \quad \operatorname{Re}(c)>\operatorname{Re}(a)>0 \\
{ }_{2} F_{1}(a, b, c, z)=\sum_{v=0}^{b-c}\left[a_{v}(c-b)_{v} /\left(c_{v} v !\right)\right](-z)^{v}(1-z)^{-a-v} \quad \text { if } \quad b-c \geq 0 \quad \text { is an integer } \\
{ }_{2} F_{1}(a, b, c, z)=\Gamma(c) \Gamma(c-b-a) \Gamma(c-b)^{-1} \Gamma(c-a)^{-1} \sum_{v=0}^{c-b-1}\left[b_{v}(b-c+1)_{v} /\left(v !(b-c+a+1)_{v}\right)\right] z^{-b-v}(z-1)^{v} \ldots \\
+(-1)^{c-b-a} \Gamma(c) \Gamma(b-c+a) \Gamma(b)^{-1} \Gamma(a)^{-1} \sum_{v=0}^{b-1}\left[(c-b)_{v}(1-b)_{v} /\left(v !(c-b-a+1)_{v}\right)\right] z^{-b-c}(z-1)^{v+c-b-a} \\
\text { if } c-b \geq 1 \text { and } b \geq 1 \text { are integers }
\end{gathered}
$$

${ }_{2} F_{1}(a, b, c, z)=\Gamma(c) \Gamma(b)^{-1} \Gamma(c-b)^{-1} \int_{0}^{1} d t t^{b-1}(1-t)^{c-b-1}(1-z t)^{-a} \quad$ if $\quad|z|<1, \quad$ and also $\quad \operatorname{Re}(b)>0, \operatorname{Re}(c-b)>0$

$$
\begin{gathered}
\int_{0}^{\infty} d r r^{M} e_{1}^{-\zeta r} F_{1}(a, c, \kappa r)=\left[\Gamma(M+1) / \zeta^{M+1}\right]_{2} F_{1}(a, M+1, c, \kappa / \zeta) \quad \text { if } \quad \operatorname{Re}(\zeta)>0 \quad \text { and } \quad M>-1 \\
\int_{0}^{\infty} d r r^{M-1} e_{1}^{-\zeta r} F_{1}(a, M, k r)_{1} F_{1}\left(a^{\prime}, M, k^{\prime} r\right)=\Gamma(M) \zeta^{a+a^{\prime}-M}(\zeta-k)^{-a}\left(\zeta-k^{\prime}\right)_{2}^{-a^{\prime}} F_{1}\left(a, a^{\prime}, M, u\right) \\
\text { where } u=k k^{\prime} /(\zeta-k)\left(\zeta-k^{\prime}\right) \quad \text { and we require } \operatorname{Re}(\zeta)>0 \quad \text { and } \quad M>0 \\
\int_{0}^{\infty} d r r^{M-1+s} e_{1}^{-\zeta r} F_{1}(a, M, k r)_{1} F_{1}\left(a^{\prime}, M, k^{\prime} r\right)=(-\partial / \partial \zeta)^{s}\left\{\Gamma(M) \zeta^{a+a^{\prime}-M}(\zeta-k)^{-a}\left(\zeta-k^{\prime}\right)_{2}^{-a^{\prime}} F_{1}\left(a, a^{\prime}, M, u\right)\right\}
\end{gathered}
$$

\section{Appendix 2: matrix elements of A $\bullet p$}

Following standard procedures [13,14], we get the following exact matrix elements for general transitions between bound states (we choose the $\mathbf{z}$ axis parallel to the radiation electric field $\mathbf{E}$ ), induced by the term linear in $\mathbf{A}(\mathbf{r})$ ( $\mathbf{A}(\mathbf{r})$ is the spatially dependent part of the vector potential operator for the radiation field) in the electron-radiation interaction:

$$
\begin{gathered}
<v \lambda \mu\left|\exp \left(i \mathbf{k}_{p h} \bullet \mathbf{r}\right) \partial / \partial z\right| n l m>=\sum_{S=0}^{\infty} \sum_{\sigma=-S}^{S}\left\{F_{\text {ang }}^{(+)} F_{\text {rad }}^{(+)}+F_{\text {ang }}^{(-)} F_{\text {rad }}^{(-)}\right\} \\
F_{\text {ang }}^{(+)}=4 \pi i^{S} Y_{S \sigma} *\left(\varepsilon_{p h}\right) \int d \Omega Y_{\lambda \mu} * Y_{S \sigma} Y_{l+1 m}[(l+1-m)(l+1+m) /(2 l+1)(2 l+3)]^{1 / 2} \quad l \geq 0 \\
F_{\text {ang }}^{(-)}=0 \quad \text { if } \quad l=0
\end{gathered}
$$




$$
=4 \pi i^{S} Y_{S \sigma} *\left(\varepsilon_{p h}\right) \int d \Omega Y_{\lambda \mu} * Y_{S \sigma} Y_{l-1 m}[(l-m)(l+m) /(2 l+1)(2 l-1)]^{1 / 2} \quad \text { if } \quad l>0
$$

The angular integrals vanish for most choices of the parameters in the spherical harmonic functions, see (eq. 2.3) below. The " $3 \mathrm{Y}$ " factor $\int d \Omega Y_{l 1 m 1} * Y_{l 2 m 2} Y_{l 3 m 3}$ vanishes exactly, unless

$$
\begin{gathered}
m_{1}+m_{2}+m_{3}=0 \\
l_{1}+l_{2}+l_{3}=\text { even integer }
\end{gathered}
$$

$l_{1}, l_{2}, l_{3}$ non-negative integers satisfying the "triangular inequalities"

$$
\begin{aligned}
& \left|l_{1}-l_{2}\right| \leq l_{3} \leq l_{1}+l_{2} \\
& \text { and }\left|l_{2}-l_{3}\right| \leq l_{1} \leq l_{2}+l_{3}
\end{aligned}
$$$$
\text { and }\left|l_{3}-l_{1}\right| \leq l_{2} \leq l_{3}+l_{1}
$$

and if any one of the three numbers is zero, the other two must be equal.

Such conditions restrict the sums over angular momentum quantum numbers very strongly. In addition, it may also happen that the Y's in the prefactors independently vanish for certain choices of the directions of incident field polarization and propagation vector $\mathbf{k}_{\mathrm{ph}}=\mathrm{k}_{p h} \varepsilon_{\mathrm{ph}}$.

In the sum in (eq. 6) it is obvious that contributions with $S>\lambda+l+1$ have null angular coefficients. For the geometry of our helium experiments in the DESY FEL Phase 1, the $z$ axis was chosen parallel to the radiant electric field, then, due to transversality, $\cos \theta=0$ and there are even less non-trivial choices of $\lambda S l$ for either $F_{\text {ang }}^{(+)}$or $F_{\text {ang }}^{(-)}$.

The radial integrals are

$$
\begin{aligned}
F_{\mathrm{rad}}^{(-)} & =\int_{0}^{\infty} r^{2} d r j_{S}\left(k_{\mathrm{ph}} r\right) f_{\mathrm{v} \lambda}(r)\left[\partial f_{n l} / \partial r+[(1+l) / r] f_{n l}\right] \\
& =N_{\mathrm{v} \lambda} N_{n l} n^{-l-1} v^{-\lambda}\left\{-\int_{0}^{\infty} r^{2} d r j_{S}\left(k_{\mathrm{ph}} r\right)(2 r)^{\lambda+l} \exp [-r(1 / n+1 / v)]_{1} F_{1}[1+\lambda-v ; 2+2 \lambda ; 2 r / v]_{1} F_{1}[1+l-n ; 2+2 l ; 2 r / n]\right. \\
\ldots & +n(1+2 l) \int_{0}^{\infty} r^{1} d r j_{S}\left(k_{\mathrm{ph}} r\right)(2 r)^{\lambda+l} \exp [-r(1 / n+1 / v)]_{1} F_{1}[1+\lambda-v ; 2+2 \lambda ; 2 r / v]_{1} F_{1}[1+l-n ; 2+2 l ; 2 r / n] \\
\ldots & \left.+[(1+l-n) /(1+l)] \int_{0}^{\infty} r^{2} d r j_{S}\left(k_{\mathrm{ph}} r\right)(2 r)^{\lambda+l} \exp [-r(1 / n+1 / v)]_{1} F_{1}[1+\lambda-\mathrm{v} ; 2+2 \lambda ; 2 r / v]_{1} F_{1}[2+l-n ; 3+2 l ; 2 r / n]\right\} \\
F_{\mathrm{rad}}^{(+)} & =\int_{0}^{\infty} r^{2} d r j_{S}\left(k_{\mathrm{ph}} r\right) f_{\mathrm{v} \lambda}(r)\left[\partial f_{n l} / \partial r-[l / r] f_{n l}\right] \\
& =N_{\mathrm{v} \lambda} N_{n l} n^{-l-1} \mathrm{v}^{-\lambda}\left\{-\int_{0}^{\infty} r^{2} d r j_{S}\left(k_{\mathrm{ph}} r\right)(2 r)^{\lambda+l} \exp [-r(1 / n+1 / v)]_{1} F_{1}[1+\lambda-\mathrm{v} ; 2+2 \lambda ; 2 r / v]_{1} F_{1}[1+l-n ; 2+2 l ; 2 r / n]\right. \\
\ldots & \left.+[(1+l-n) /(1+l)] \int_{0}^{\infty} r^{2} d r j_{S}\left(k_{\mathrm{ph}} r\right)(2 r)^{\lambda+l} \exp [-r(1 / n+1 / v)]_{1} F_{1}[1+\lambda-v ; 2+2 \lambda ; 2 r / v]_{1} F_{1}[2+l-n ; 3+2 l ; 2 r / n]\right\}
\end{aligned}
$$

All integrals in $F_{\text {rad }}$ can be expressed exactly in terms of standard hypergeometric functions ${ }_{2} F_{1}$ :

$$
\begin{aligned}
F_{\text {rad }}^{(+)}= & (--1 / n) N_{v \lambda} N_{n l}\left(k_{p h}\right)^{S}[(2 S+1) ! !]^{-1} \sum_{u=0}^{(v-\lambda-1)} \sum_{v=0}^{(n-l-1)}(2 / v)^{u+\lambda}(2 / n)^{v+l} \ldots \\
\ldots \quad & {\left[(1+\lambda-v)_{u} /\left[u !(2+2 \lambda)_{u}\right]\right]\left[(1+l-n)_{v} /\left[v !(2+2 l)_{v}\right]\right] \Gamma(M+1) \zeta_{2}^{-M-1} F_{1}\left(1+S ; M+1 ; 2+2 S ; 2 i k_{p h} / \zeta\right) \ldots } \\
\ldots+ & {[(1+l-n) /(n+n l)] N_{v \lambda} N_{n l}\left(k_{p h}\right)^{S}[(2 S+1) ! !]^{-1} \sum_{u=0}^{(v-\lambda-1)} \sum_{v=0}^{(n-l-2)}(2 / v)^{u+\lambda}(2 / n)^{v+l} \ldots } \\
\ldots \quad & {\left[(1+\lambda-v)_{u} /\left[u !(2+2 \lambda)_{u}\right]\right]\left[(2+l-n)_{v} /\left[v !(3+2 l)_{v}\right]\right] \Gamma(M+1) \zeta_{2}^{-M-1} F_{1}\left(1+S ; M+1 ; 2+2 S ; 2 i k_{p h} / \zeta\right) } \\
M \equiv & 2+\lambda+l+S+u+v \\
\zeta \equiv & 1 / v+1 / n+i k_{p h} \\
F_{\text {rad }}^{(-)}= & (--1 / n) N_{v \lambda} N_{n l}\left(k_{p h}\right)^{S}[(2 S+1) ! !]^{-1} \sum_{u=0}^{(v-\lambda-1)} \sum_{v=0}^{(n-l-1)}(2 / v)^{u+\lambda}(2 / n)^{v+l} \ldots \\
\ldots \quad & {\left[(1+\lambda-v)_{u} /\left[u !(2+2 \lambda)_{u}\right]\right]\left[(1+l-n)_{v} /\left[v !(2+2 l)_{v}\right]\right] \Gamma(M+1) \zeta_{2}^{-M-1} F_{1}\left(1+S ; M+1 ; 2+2 S ; 2 i k_{p h} / \zeta\right) \ldots } \\
\ldots+ & (1+2 l) N_{v \lambda} N_{n l}\left(k_{p h}\right)^{S}[(2 S+1) ! !]^{-1} \sum_{u=0}^{(v-\lambda-1)} \sum_{v=0}^{(n-l-1)}(2 / v)^{u+\lambda}(2 / n)^{v+l} \ldots \\
& {\left[(1+\lambda-v)_{u} /\left[u !(2+2 \lambda)_{u}\right]\right]\left[(1+l-n)_{v} /\left[v !(2+2 l)_{v}\right]\right] \Gamma(M) \zeta_{2}^{-M} F_{1}\left(1+S ; M ; 2+2 S ; 2 i k_{p h} / \zeta\right) \ldots } \\
\ldots+ & {[(1+l-n) /(n+n l)] N_{v \lambda} N_{n l}\left(k_{p h}\right)^{S}[(2 S+1) ! !]^{-1} \sum_{u=0}^{(v-\lambda-1)} \sum_{v=0}^{(n-l-2)}(2 / v)^{u+\lambda}(2 / n)^{v+l} \ldots } \\
\ldots \quad & {\left[(1+\lambda-v)_{u} /\left[u !(2+2 \lambda)_{u}\right]\right]\left[(2+l-n)_{v} /\left[v !(3+2 l)_{v}\right]\right] \Gamma(M+1) \zeta_{2}^{-M-1} F_{1}\left(1+S ; M+1 ; 2+2 S ; 2 i k_{p h} / \zeta\right) }
\end{aligned}
$$


For the simplest case of interest $\mathrm{v}=2, \lambda=1, n=1, l=0$ only the terms $S=0,2$ survive. They reduce to the elementary result $\left[-(2 / 3)^{4} \sqrt{ } 2\right]$ in the electric dipole approximation $k_{p h}=0$.

Similarly, for transitions from a bound into a quasi-free state, we get

$$
\begin{aligned}
& <\mathbf{k}_{e}\left|\exp \left(i \mathbf{k}_{p h} \bullet \mathbf{r}\right) \partial / \partial z\right| n l m>=\sum_{\lambda=0}^{\infty} \sum_{\mu=-\lambda}^{\lambda} \sum_{S=0}^{\infty} \sum_{\sigma=-S}^{S}\left\{G_{\text {ang }}^{(+)} G_{\text {rad }}^{(+)}+G_{\text {ang }}^{(-)} G_{\text {rad }}^{(-)}\right\} \\
& G_{\text {ang }}^{(+)}=4 \pi(-1)^{\lambda} i^{S} Y_{\lambda \mu}\left(\varepsilon_{k e}\right) Y_{S \sigma} *\left(\varepsilon_{p h}\right) \int d \Omega Y_{\lambda \mu} * Y_{S \sigma} Y_{l+1 m}[(l+1-m)(l+1+m) /(2 l+1)(2 l+3)]^{1 / 2} \quad l \geq 0 \\
& G_{\text {ang }}^{(-)}=0 \quad \text { if } \quad l=0 \\
& =4 \pi(-1)^{\lambda} i^{S} Y_{\lambda \mu}\left(\varepsilon_{k e}\right) Y_{S \sigma} *\left(\varepsilon_{p h}\right) \int d \Omega Y_{\lambda \mu} * Y_{S \sigma} Y_{l-1 m}[(l-m)(l+m) /(2 l+1)(2 l-1)]^{1 / 2} \quad \text { if } \quad l>0
\end{aligned}
$$

( $\varepsilon_{k e}$ along $z, \varepsilon_{p h}$ orthogonal to $z$ ).

For the radial integrals, case bound-free transitions,

$$
\begin{aligned}
& G_{\mathrm{rad}}^{(+)}= \int_{0}^{\infty} r^{2} d r\left(2 i k_{e} r\right)^{\lambda} \exp \left[-i k_{e} r\right]_{1} F_{1}\left[1+\lambda+i / k_{e} ; 2+2 \lambda ; 2 i k_{e} r\right] j_{S}\left(k_{p h} r\right)\left[\partial f_{n l} / \partial r-[l / r] f_{n l}\right] \\
&= C\left(k_{e}, \lambda\right) N_{n l} n^{-l-1}\left\{-i n t_{0}^{\infty} r^{2} d r j_{S}\left(k_{p h} r\right)\left(2 k_{e} r\right)^{\lambda}(2 r)^{l} \exp \left[-r\left(1 / n+i k_{e}\right)\right]\right. \\
&{ }_{1} F_{1}\left[1+\lambda+i / k_{e} ; 2+2 \lambda ; 2 i k_{e} r\right]_{1} F_{1}[1+l-n ; 2+2 l ; 2 r / n] \\
& \ldots+ {[(1+l-n) /(1+l)] \int_{0}^{\infty} r^{2} d r j_{S}\left(k_{p h} r\right)\left(2 k_{e} r\right)^{\lambda}(2 r)^{l} \exp \left[-r\left(1 / n+i k_{e}\right)\right] } \\
&\left.{ }_{1} F_{1}\left[1+\lambda+i / k_{e} ; 2+2 \lambda ; 2 i k_{e} r\right]_{1} F_{1}[2+l-n ; 3+2 l ; 2 r / n]\right\} \\
& G_{\mathrm{rad}}^{(-)}= \int_{0}^{\infty} r^{2} d r\left(2 i k_{e} r\right)^{\lambda} \exp \left[-i k_{e} r\right] \\
&{ }_{1} F_{1}\left[1+\lambda+i / k_{e} ; 2+2 \lambda ; 2 i k_{e} r\right]_{S}\left(k_{p h} r\right)\left[\partial f_{n l} / \partial r+[(1+l) / r] f_{n l}\right] \\
&= C\left(k_{e}, \lambda\right) N_{n l} n^{-l-1}\left\{-\int_{0}^{\infty} r^{2} d r j_{S}\left(k_{p h} r\right)\left(2 k_{e} r\right)^{\lambda}(2 r)^{l} \exp \left[-r\left(1 / n+i k_{e}\right)\right]\right. \\
&{ }_{1} F_{1}\left[1+\lambda+i / k_{e} ; 2+2 \lambda ; 2 i k_{e} r\right]_{1} F_{1}[1+l-n ; 2+2 l ; 2 r / n] \\
& \ldots n(1+2 l) \int_{0}^{\infty} r^{1} d r j_{S}\left(k_{p h} r\right)\left(2 k_{e} r\right)^{\lambda}(2 r)^{l} \exp \left[-r\left(1 / n+i k_{e}\right)\right] \\
&{ }_{1} F_{1}\left[1+\lambda+i / k_{e} ; 2+2 \lambda ; 2 i k_{e} r\right]_{1} F_{1}[1+l-n ; 2+2 l ; 2 r / n] \\
&+ {[(1+l-n) /(1+l)] \int_{0}^{\infty} r^{2} d r j_{S}\left(k_{p h} r\right)\left(2 k_{e} r\right)^{\lambda}(2 r)^{l} \exp \left[-r\left(1 / n+i k_{e}\right)\right] } \\
&\left.{ }_{1} F_{1}\left[1+\lambda+i / k_{e} ; 2+2 \lambda ; 2 i k_{e} r\right]_{1} F_{1}[2+l-n ; 3+2 l ; 2 r / n]\right\}
\end{aligned}
$$

These integrals no longer have exact analytic expressions in terms of elementary special functions. They can, however, be written in terms of some integrals over the interval $(0,1)$ which are easy to calculate numerically, and which reduce to some ${ }_{2} F_{1}$ in the special cases $\alpha=0, q=0$, or $\alpha-M-1-q=0$, or $x=0$ or $y=0$ :

$$
\begin{aligned}
& G_{\mathrm{rad}}^{(+)}=C\left(k_{e}, \lambda\right) N_{n l}\left(2 i k_{e}\right)^{\lambda}\left(k_{p h}\right)^{S}(2 / n)^{l}[(2 S+1) ! !]^{-1} \ldots \\
& \ldots \quad\left\{(-1 / n) \sum_{v=0}^{n-l-1}\left[(1+l-n)_{v} /\left(v !(2+2 l)_{v}\right)\right](2 / n)^{v} \quad \Gamma(1+M) \ldots\right. \\
& \ldots \quad \sum_{q=0}^{1+M-\Lambda}\left[(\Lambda-M-1)_{q}(\alpha)_{q} /\left(q !(\Lambda)_{q}\right)\right]\left(-2 i k_{e}\right)^{q}\left(\zeta-2 i k_{e}\right)^{-\alpha-q} \zeta^{\alpha-M-1} \Upsilon(S) \int_{0}^{1} d t t^{S}(1-t)^{S}(1-x t)^{\alpha-M-1-q}(1-y t)^{-\alpha} \\
& +(1+l-n)(n+n l)^{-1} \sum_{v=0}^{n-l-2}\left[(2+l-n)_{v} /\left(v !(3+2 l)_{v}\right)\right](2 / n)^{v} \Gamma(1+M) \ldots \\
& \left.\ldots \quad \sum_{q=0}^{1+M-\Lambda}\left[(\Lambda-M-1)_{q}(\alpha)_{q} /\left(q !(\Lambda)_{q}\right)\right]\left(-2 i k_{e}\right)^{q}\left(\zeta-2 i k_{e}\right)^{-\alpha-q \zeta^{\alpha-M-1} \Upsilon(S)} \int_{0}^{1} d t t^{S}(1-t)^{S}(1-x t)^{\alpha-M-1-q}(1-y t)^{-\alpha}\right\}
\end{aligned}
$$


with

$$
\begin{aligned}
& \Upsilon(S)=\Gamma(2+2 S)[\Gamma(1+S)]^{-2} \\
& M=2+\lambda+l+S+v \text { (notice that this depends on } v \text {, a summation index) } \\
& {[\Lambda=2+2 \lambda]} \\
& \alpha=1+\lambda+i / k_{e} \\
& \zeta=1 / n+i k_{e}+i k_{p h} \quad x=2 i k_{p h} / \zeta \quad y=2 i k_{p h} /\left(\zeta-2 i k_{e}\right) \\
& G_{\mathrm{rad}}^{(-)}=C\left(k_{e}, \lambda\right) N_{n l}\left(2 i k_{e}\right)^{\lambda}\left(k_{p h}\right)^{S}(2 / n)^{l}[(2 S+1) ! !]^{-1} \ldots \\
& \ldots \quad\left\{(-1 / n) \sum_{v=0}^{n-l-1}\left[(1+l-n)_{v} /\left(v !(2+2 l)_{v}\right)\right](2 / n)^{v} \Gamma(1+M)\left(\zeta-2 i k_{e}\right)^{-\alpha} \ldots\right.
\end{aligned}
$$

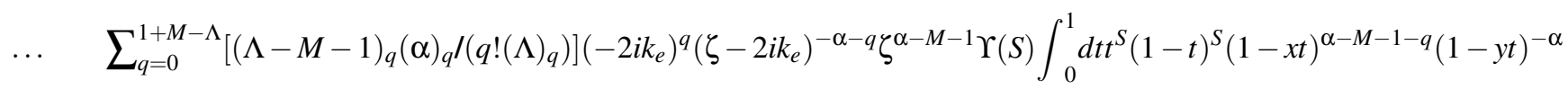

$$
\begin{aligned}
& +(1+2 l) \sum_{v=0}^{n-l-1}\left[(1+l-n)_{v} /\left(v !(2+2 l)_{v}\right)\right](2 / n)^{v} \Gamma(M)\left(\zeta-2 i k_{e}\right)^{-\alpha} \ldots
\end{aligned}
$$

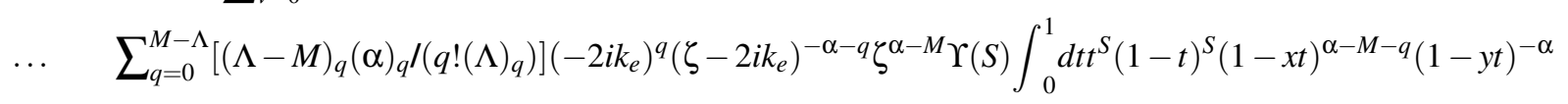

$$
\begin{aligned}
& +(1+l-n)(n+n l)^{-1} \sum_{v=0}^{n-l-2}\left[(2+l-n)_{v} /\left(v !(3+2 l)_{v}\right)\right](2 / n)^{v} \quad \Gamma(1+M)\left(\zeta-2 i k_{e}\right)^{-\alpha} \ldots \\
& \ldots \quad \sum_{q=0}^{1+M-\Lambda}\left[(\Lambda-M-1)_{q}(\alpha)_{q} /\left(q !(\Lambda)_{q}\right)\right]\left(-2 i k_{e}\right)^{q}\left(\zeta-2 i k_{e}\right)^{-\alpha-q} \zeta^{\alpha-M-1} \Upsilon(S) \\
& \left.\int_{0}^{1} d t t^{S}(1-t)^{S}(1-x t)^{\alpha-M-1-q}(1-y t)^{-\alpha}\right\}
\end{aligned}
$$

Finally, we discuss transitions into unbound $\mathbf{k}$ ' from unbound $\mathbf{k}$ states.

Let us start by saying that if one uses plane waves $\exp (-i \mathbf{k} \bullet \mathbf{r})$ to describe the unbound electron states, 1-photon absorption is strictly forbidden due to conservation of energy and linear momentum. In fact, the matrix element of p.A is in this case proportional to $\delta\left(\mathbf{k}_{2}-\mathbf{k}_{1}+\mathbf{k}_{\text {photon }}\right)$. Typical values for experiments at the DESY FEL Phase 1 would be $k_{\text {photon }}=0.00035, k_{1}=$ $0.35, k_{2}=1.05$, all in units of $r_{\mathrm{Bohr}}^{-1}$. It is clear there is no way to make the argument of the $\delta$-function vanish.

If, however, we consider exact Coulomb states (unbound electron in the presence of a positive ion, eq. 1.2 or eq. 1.3) we find a rather large value for the matrix element, comparable to values for 1-photon absorption in the transitions (ground state)? (excited bound state).

The exact matrix element can be written as an infinite series only, but for parameter values of current interest, the series converges very rapidly. The result, assuming the radiant field $\mathbf{E}$ is parallel to $\mathbf{z}$, and using the definitions below is,

$$
\begin{aligned}
\alpha & =1+l+i / k \\
\alpha^{\prime} & =1+l^{\prime}+i / k^{\prime} \\
\Lambda & =2+2 l \\
\Lambda^{\prime} & =2+2 l^{\prime} \\
\varepsilon & =\mathbf{k} / k \\
\varepsilon^{\prime} & =\mathbf{k}^{\prime} / k^{\prime} \\
\varepsilon_{\mathrm{ph}} & =\mathbf{k}_{\mathrm{ph}} / k_{\mathrm{ph}}
\end{aligned}
$$

$$
\begin{array}{r}
<\mathbf{k}\left|\exp \left(i \mathbf{k}_{\mathrm{ph}} \bullet \mathbf{r}\right) \partial / \partial z\right| \mathbf{k}>=\sum_{l m} \sum_{l^{\prime} m^{\prime}} \sum_{S \sigma} H_{a n g}^{(+)}\left(l^{\prime} m^{\prime} \varepsilon^{\prime}, S \sigma \varepsilon_{\mathrm{ph}}, \operatorname{lm} \varepsilon\right) H_{r a d}^{(+)}\left(l^{\prime} k^{\prime}, S k_{\mathrm{ph}}, l k\right) \ldots \\
\ldots+\sum_{l m} \sum_{l^{\prime} m^{\prime}} \sum_{S \sigma} H_{a n g}^{(-)}\left(l^{\prime} m^{\prime} \varepsilon^{\prime}, S \sigma \varepsilon_{\mathrm{ph}}, l m \varepsilon\right) H_{r a d}^{(-)}\left(l^{\prime} k^{\prime}, S k_{\mathrm{ph}}, l k\right)
\end{array}
$$




$$
\begin{aligned}
& H_{\text {ang }}^{(+)}\left(l^{\prime} m^{\prime} \varepsilon^{\prime}, S \sigma \varepsilon_{\mathrm{ph}}, l m \varepsilon\right)=4 \pi i^{S} Y_{l^{\prime} m^{\prime}}\left(\varepsilon^{\prime}\right) Y *_{S \sigma}\left(\varepsilon_{\mathrm{ph}}\right) Y_{l m} *(\varepsilon)\left[(l+1+m)(l+1-m)(2 l+1)^{-1}(2 l+3)^{-1}\right]^{1 / 2} \ldots
\end{aligned}
$$

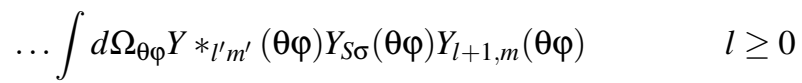

$$
\begin{aligned}
& H_{\text {ang }}^{(-)}\left(l^{\prime} m^{\prime} \varepsilon^{\prime}, S \sigma \varepsilon_{\mathrm{ph}}, l m \varepsilon\right)=0 \quad \text { if } \quad l=0 \\
& =4 \pi i^{S} Y_{l^{\prime} m^{\prime}}\left(\varepsilon^{\prime}\right) Y *_{S \sigma}\left(\varepsilon_{\mathrm{ph}}\right) Y_{l m} *(\varepsilon)\left[(l+m)(l-m)(2 l+1)^{-1}(2 l-1)^{-1}\right]^{1 / 2} \ldots \\
& \ldots \int d \Omega_{\theta \varphi} Y_{l^{\prime} m^{\prime}} *(\theta \varphi) Y_{S \sigma}(\theta \varphi) Y_{l-1, m}(\theta \varphi) \quad \text { if } \quad l>0
\end{aligned}
$$

$$
\begin{aligned}
H_{\mathrm{rad}}^{(+)}\left(l^{\prime} k^{\prime}, S k_{\mathrm{ph}}, l k\right)= & C(k, l) C\left(k^{\prime}, l^{\prime}\right)(2 i k)^{l}\left(2 i k^{\prime}\right)^{l^{\prime}} \ldots \\
& \ldots\left\{(-i k) \int_{0}^{\infty} r^{2+l+l^{\prime}} d r \exp \left[-i\left(k+k^{\prime}\right) r\right] j_{S}\left(k_{\mathrm{ph}} r\right)_{1} F_{1}\left(\alpha^{\prime}, \Lambda^{\prime}, 2 i k^{\prime} r\right)_{1} F_{1}(\alpha, \Lambda, 2 i k r) \ldots\right. \\
& \left.\ldots+\left[i k-(1+l)^{-1}\right] \int_{0}^{\infty} r^{2+l+l \prime} d r \exp \left[-i\left(k+k^{\prime}\right) r\right] j_{S}\left(k_{p h} r\right)_{1} F_{1}\left(\alpha^{\prime}, \Lambda^{\prime}, 2 i k^{\prime} r\right)_{1} F_{1}(\alpha+1, \Lambda+1,2 i k r)\right\} \\
H_{\mathrm{rad}}^{(-)}\left(l^{\prime} k^{\prime}, S k_{\mathrm{ph}}, l k\right)= & C(k, l) C\left(k^{\prime}, l^{\prime}\right)(2 i k)^{l}\left(2 i k^{\prime}\right)^{l^{\prime}} \ldots \\
& \ldots\left\{(1+2 l) \int_{0}^{\infty} r^{1+l+l^{\prime}} d r \exp \left[-i\left(k+k^{\prime}\right) r\right] j_{\lambda}\left(k_{\mathrm{ph}} r\right)_{1} F_{1}\left(\alpha^{\prime}, \Lambda^{\prime}, 2 i k^{\prime} r\right)_{1} F_{1}(\alpha, \Lambda, 2 i k r) \ldots\right. \\
& \ldots(-i k) \int_{0}^{\infty} r^{2+l+l^{\prime}} d r \exp \left[-i\left(k+k^{\prime}\right) r\right] j_{\lambda}\left(k_{\mathrm{ph}} r\right)_{1} F_{1}\left(\alpha^{\prime}, \Lambda^{\prime}, 2 i k^{\prime} r\right)_{1} F_{1}(\alpha, \Lambda, 2 i k r) \ldots \\
\ldots+ & {\left.\left[i k-(1+l)^{-1}\right] \int_{0}^{\infty} r^{2+l+l^{\prime}} d r \exp \left[-i\left(k+k^{\prime}\right) r\right] j_{\lambda}\left(k_{\mathrm{ph}} r\right)_{1} F_{1}\left(\alpha^{\prime}, \Lambda^{\prime}, 2 i k^{\prime} r\right)_{1} F_{1}(\alpha+1, \Lambda+1,2 i k r)\right\} }
\end{aligned}
$$

The expressions for transitions with bound or unbound initial or final states have all the same form, and can be obtained formally from each other by replacement of $\mathrm{N}_{n l}$ and $1 / \mathrm{n}$ occurring for bound states, with $\mathrm{C}(\mathrm{k}, l)$ and $i \mathrm{k}$, occurring for unbound states. Here, however, the ${ }_{1} \mathrm{~F}_{1}$ do not, in general, reduce to polynomials, because the arguments $\alpha \alpha^{\prime}$ are not negative integers. These integrals must be handled with care.

The radial integrals are all, generally speaking, non-vanishing. On the other hand, in the angular functions we have "3 Y" integrals which vanish for most values of the arguments, in particular for $S>l+1+l^{\prime}$ (in $H_{\text {ang }}^{(+)}$) and for $S>l-1+l^{\prime}\left(\right.$ in $H_{\text {ang }}^{(-)}$).

For the radial integrals corresponding to non-zero angular integrals, we might use the spherical Bessel function expansion (eq. 1.5c) which is a finite sum of inverse powers $\left(k_{p h} r\right)^{-q}, 1 \leq q \leq S+1$. Notice that, given the restrictions $0 \leq S \leq l+l^{\prime}+1$ (for $H_{\mathrm{rad}}^{(+)}$), $0 \leq S \leq l+l^{\prime}-1$ (for $H_{\mathrm{rad}}^{(+)}$) just mentioned, the integrand will never be singular at the origin. In addition, we use the integral representation (eq. 1.7b) for ${ }_{1} F_{1}(\alpha, \Lambda, 2 i k r)$ plus (eq. 1.7f). We can also use the infinite power series representation, (eq. 1.5b).

From a practical point of view, however, the development into exponentials is numerically inefficient, because the result depends on differences of large quantities which are almost the same. In addition, it is not easily applicable to the limit $\mathrm{k}_{\mathrm{ph}}=0$ because of the factors $k_{\mathrm{ph}}^{-q}, 1 \leq q \leq S+1$. We have, however, calculated some of these integrals for purposes of checking. The calculations take about 10 times longer than using the power series expansion (eq. 1.5b) of the spherical Bessel function (discussed below) and precision is not as good. But the results agree to within 1 part in $10^{5}$, and show that the general formulae are not in error.

For actual calculations we use the power series expansion (eq. 1.5b) for the spherical Bessel function, truncating the series at some adequate $Q_{\max }$, which will depend on $k_{\mathrm{ph}}$. 


$$
\begin{aligned}
H_{\mathrm{rad}}^{(+)}= & C(k, l) C\left(k^{\prime}, l^{\prime}\right)(2 i k)^{l}\left(2 i k^{\prime}\right)^{l^{\prime}} \sum_{q=0}^{Q \max } k_{\mathrm{ph}}^{2 q+S} b_{q}^{(S)} \\
& \left\{(-i k) \Gamma(M+1) \Gamma(\Lambda) \Gamma(\alpha)^{-1} \Gamma(\Lambda-\alpha)^{-1} \int_{0}^{1} d t t^{\alpha-1}(1-t)^{\Lambda-\alpha-1}(\zeta-2 i k t)_{2}^{-M-1} F_{1}\left(\alpha^{\prime}, M+1, \Lambda^{\prime}, z\right) \ldots\right. \\
+ & {\left.\left[i k-(1+l)^{-1}\right] \Gamma(M+1) \Gamma(\Lambda) \Gamma(\alpha)^{-1} \Gamma(\Lambda-\alpha)^{-1} \int_{0}^{1} d t t^{\alpha-1}(1-t)^{\Lambda-\alpha-1}(\zeta-2 i k t)_{2}^{-M-1} F_{1}\left(\alpha^{\prime}+1, M+1, \Lambda^{\prime}+1, z\right)\right\} } \\
H_{\mathrm{rad}}^{(-)}= & C(k, l) C\left(k^{\prime}, l^{\prime}\right)(2 i k)^{l}\left(2 i k^{\prime}\right)^{l^{\prime}} \sum_{q=0}^{Q m a x} k_{\mathrm{ph}}^{2 q+S} b_{q}^{(S)} \\
& \left\{(1+2 l) \Gamma(M) \Gamma(\Lambda) \Gamma(\alpha)^{-1} \Gamma(\Lambda-\alpha)^{-1} \int_{0}^{1} d t t^{\alpha-1}(1-t)^{\Lambda-\alpha-1}(\zeta-2 i k t)_{2}^{-M} F_{1}\left(\alpha^{\prime}, M, \Lambda^{\prime}, z\right)\right. \\
& (-i k) \Gamma(M+1) \Gamma(\Lambda) \Gamma(\alpha)^{-1} \Gamma(\Lambda-\alpha)^{-1} \int_{0}^{1} d t t^{\alpha-1}(1-t)^{\Lambda-\alpha-1}(\zeta-2 i k t)_{2}^{-M-1} F_{1}\left(\alpha^{\prime}, M+1, \Lambda^{\prime}, z\right) \ldots \\
+ & {\left.\left[i k-(1+l)^{-1}\right] \Gamma(M+1) \Gamma(\Lambda) \Gamma(\alpha)^{-1} \Gamma(\Lambda-\alpha)^{-1} \int_{0}^{1} d t t^{\alpha-1}(1-t)^{\Lambda-\alpha-1}(\zeta-2 i k t)_{2}^{-M-1} F_{1}\left(\alpha^{\prime}+1, M+1, \Lambda^{\prime}+1, z\right)\right\} }
\end{aligned}
$$

where, now, we assume $k<k^{\prime}$ in order to avoid singular integrands, and define

$$
\begin{aligned}
M & =2+l+l^{\prime}+2 q+S \\
\zeta & =i k+i k^{\prime} \\
z & =2 i k^{\prime} /(\zeta-2 i k t)
\end{aligned}
$$

Depending on the exponents, the integrand may result ill-conditioned at the end points $t=0$ and $t=1$. Then we truncate the integration, i.e, we integrate in the interval $\varepsilon<t<1-\varepsilon$, with $\varepsilon \sim 10^{-7}$.

\section{Appendix 3: Matrix elements of $A \bullet A$}

Now we discuss the matrix elements $<$ final $\left|\exp \left(2 i \mathbf{k}_{p h} \bullet \mathbf{r}\right)\right|$ initial $>$, where final and initial can be bound or unbound energy eigenstates, with $E_{\text {final }}>E_{\text {initial }}$.

If $k_{\mathrm{ph}}=0$ they vanish identically because eigenstates with different energy are orthogonal. We are, of course, interested in the general case $k_{\mathrm{ph}}>0$.

For 2-photon bound-bound transitions we have

$$
\begin{aligned}
<v \lambda \mu\left|\exp \left(2 i \mathbf{k}_{\mathrm{ph}} \bullet \mathbf{r}\right)\right| n l m> & =\int d r r^{2} d \Omega f_{\mathrm{v} \lambda}(r) Y_{\lambda \mu} *(\theta \varphi) \exp \left(2 i \mathbf{k}_{\mathrm{ph}} \bullet \mathbf{r}\right) f_{n l}(r) Y_{l m}(\theta \varphi) \\
& =\sum_{S \sigma} 4 \pi i^{S} Y_{S \sigma} *\left(\varepsilon_{\mathrm{ph}}\right) \int d \Omega Y_{\lambda \mu} *(\theta \varphi) Y_{S \sigma}(\theta \varphi) Y_{l m}(\theta \varphi) \int d r r^{2} f_{\mathrm{v} \lambda}(r) j_{S}\left(2 k_{\mathrm{ph}} r\right) f_{n l}(r) \\
& =\sum_{S \sigma} I_{\mathrm{ang}}^{(b-b)} I_{\text {rad }}^{(b-b)} \\
I_{\mathrm{ang}}^{(b-b)} & =4 \pi i^{S} Y_{S \sigma} *\left(\varepsilon_{\mathrm{ph}}\right) \int d \Omega Y_{\lambda \mu} *(\theta \varphi) Y_{S \sigma}(\theta \varphi) Y_{l m}(\theta \varphi) \\
I_{\mathrm{rad}}^{(b-b)} & =\int d r r^{2} f_{\mathrm{v} \lambda}(r) j_{S}\left(2 k_{\mathrm{ph}} r\right) f_{n l}(r)
\end{aligned}
$$

The "3Y" angular integral has already been discussed. Using expansion (eq. 1.5a) for $j_{S}(u)$ and the definition (eq. 1.1) of the bound state radial functions,

$$
\begin{aligned}
I_{\mathrm{rad}}^{(b-b)}= & N_{\mathrm{v} \lambda} N_{n l}(2 / n)^{l}(2 / \mathrm{v})^{\lambda}\left[\left(2 k_{\mathrm{ph}}\right)^{S} /(2 S+1) ! !\right] \int d r r^{2+l+\lambda+S}\left\{\exp (-r / v){ }_{1} F_{1}(1+\lambda-v, 2+2 \lambda, 2 r / v)\right\} \cdots \\
& \ldots\left\{\exp \left(-2 i k_{p h} r\right)_{1} F_{1}\left(1+S, 2+2 S, 4 i k_{\mathrm{ph}} r\right)\right\}\left\{\exp (-r / n)_{1} F_{1}(1+l-n, 2+2 l, 2 r / n)\right\}
\end{aligned}
$$


The ${ }_{1} F_{1}$ related to the bound states are always polynomials in $\mathrm{r}$. Inserting the appropriate sums and then using the integral in (eq. 1.7f), we get

$$
\begin{aligned}
I_{\mathrm{rad}}^{(b-b)}= & N_{v \lambda} N_{n l}(2 / n)^{l}(2 / \mathrm{v})^{\lambda}\left[\left(2 k_{\mathrm{ph}}\right)^{S} /(2 S+1) ! !\right] \ldots \\
& \ldots \sum_{u=0}^{v-\lambda-1} \sum_{v=0}^{n-l-1}\left[(2 / v)^{u}(1+\lambda-v)_{u} / u !(2+2 \lambda)_{u}\right]\left[(2 / n)^{v}(1+l-n)_{v} / v !(2+2 l)_{v}\right] \ldots \\
& \ldots \Gamma(M+1) \zeta_{2}^{-M-1} F_{1}\left(1+S, M+1,2+2 S, 4 i k_{\mathrm{ph}} / \zeta\right) \\
M= & 2+l+\lambda+S+u+v \\
\zeta= & 1 / n+1 / v+2 i k_{\mathrm{ph}}
\end{aligned}
$$

The possible values of $\lambda S l$ are restricted to $|\lambda-l| \leq S \leq \lambda+l$ with $\lambda+S+l=$ even due to the angular integral. Similarly, we have for a 2-photon bound-free transition

$$
\begin{aligned}
&<\mathbf{k}^{\prime}\left|\exp \left(2 i \mathbf{k}_{\mathrm{ph}} \bullet \mathbf{r}\right)\right| n l m>=\int d r r^{2} d \Omega \Psi_{k^{\prime}} *(r \theta \varphi) \exp \left(2 i \mathbf{k}_{\mathrm{ph}} \bullet \mathbf{r}\right) f_{n l}(r) Y_{l m}(\theta \varphi) \\
&=\sum_{\lambda \mu} Y_{\lambda \mu}\left(\varepsilon^{\prime}\right) \sum_{S \sigma} 4 \pi i^{S} Y_{S \sigma} *\left(\varepsilon_{\mathrm{ph}}\right) \int d \Omega Y_{\lambda \mu} *(\theta \varphi) Y_{S \sigma}(\theta \varphi) Y_{l m}(\theta \varphi) \int d r r^{2} \Psi_{v \lambda}(r) j_{S}\left(2 k_{\mathrm{ph}} r\right) f_{n l}(r) \\
&=\sum_{\lambda \mu} \sum_{S \sigma} I_{a n g}^{(b-b)} I_{\text {rad }}^{(b-b)} \\
& I_{\text {ang }}^{(b-\text { free })}=4 \pi i^{S} Y_{\lambda \mu}\left(\varepsilon^{\prime}\right) Y_{S \sigma} *\left(\varepsilon_{\mathrm{ph}}\right) \int d \Omega Y_{\lambda \mu} *(\theta \varphi) Y_{S \sigma}(\theta \varphi) Y_{l m}(\theta \varphi) \\
& I_{\text {rad }}^{(b-\text { free })}=\int d r r^{2} \Psi_{\mathrm{v} \lambda}(r) j_{S}\left(2 k_{\mathrm{ph}} r\right) f_{n l}(r) \\
& I_{\text {rad }}^{(b-\text { free })}=C\left(k^{\prime}, \lambda\right) N_{n l}(2 / n)^{l}\left(2 i k^{\prime}\right)^{\lambda}\left[\left(2 k_{\mathrm{ph}}\right)^{S} /(2 S+1) ! !\right] \int d r r^{2+l+\lambda+S} \exp \left(-r / n-i k^{\prime} r-2 i k_{\mathrm{ph}} r\right) \ldots \\
& \ldots{ }_{1} F_{1}\left(1+\lambda+i / k^{\prime}, 2+2 \lambda, 2 i k^{\prime} r\right)_{1} F_{1}\left(1+S, 2+2 S, 4 i k_{\mathrm{ph}} r\right)_{1} F_{1}(1+l-n, 2+2 l, 2 r / n)
\end{aligned}
$$

The last ${ }_{1} F_{1}$ factor is still a polynomial in $(2 r / n)$ and we develop it:

$$
\begin{aligned}
I_{\text {rad }}^{(b-\text { free })}= & C\left(k^{\prime}, \lambda\right) N_{n l}(2 / n)^{l}\left(2 i k^{\prime}\right)^{\lambda}\left[\left(2 k_{\mathrm{ph}}\right)^{S} /(2 S+1) ! !\right] \sum_{v=0}^{n-l-1}\left[(2 / n)^{v}(1+l-n)_{v} / v !(2+2 l)_{v}\right] \ldots \\
& \ldots \int d r r^{2+l+\lambda+S} \exp \left(-r / n-i k^{\prime} r-2 i k_{\mathrm{ph}} r\right)_{1} F_{1}\left(1+\lambda+i / k^{\prime}, 2+2 \lambda, 2 i k^{\prime} r\right)_{1} F_{1}\left(1+S, 2+2 S, 4 i k_{\mathrm{ph}} r\right)
\end{aligned}
$$

In some special cases, the remaining radial integral can be written as a closed analytical expression [13].

In general, the integral is calculated numerically. We replace one of the ${ }_{1} F_{1}$ factors with its integral representation and then integrate in dr, to get

$$
\begin{aligned}
& \int d r r^{2+l+\lambda+S+v} \exp \left(-r / n-i k^{\prime} r-2 i k_{\mathrm{ph}} r\right)_{1} F_{1}\left(1+\lambda+i / k^{\prime}, 2+2 \lambda, 2 i k^{\prime} r\right)_{1} F_{1}\left(1+S, 2+2 S, 4 i k_{\mathrm{ph}} r\right)= \\
= & \Gamma(2+2 S) \Gamma(1+S)^{-2} M ! \int_{0}^{1} d t t^{S}(1-t)^{S}\left(\zeta+4 i k_{\mathrm{ph}} t\right)_{2}^{-M-1} F_{1}\left(1+\lambda+i / k^{\prime}, M+1,2+2 \lambda, 2 i k^{\prime} /\left(\zeta+4 i k_{\mathrm{ph}} t\right)\right) \\
M= & 2+l+\lambda+S+v \\
\zeta= & 1 / n+i k^{\prime}+2 i k_{\mathrm{ph}}
\end{aligned}
$$

Finally, for 2-photon free-free transitions we have 


$$
\begin{aligned}
& <\mathbf{k}^{\prime}\left|\exp \left(2 i \mathbf{k}_{\mathrm{ph}} \bullet \mathbf{r}\right)\right| \mathbf{k}>=\int d r r^{2} d \Omega \Psi_{k^{\prime}}^{*}(r \theta \varphi) \exp \left(2 i \mathbf{k}_{\mathrm{ph}} \bullet \mathbf{r}\right) \Psi_{k}(r \theta \varphi) \\
& =\sum_{\lambda \mu} Y_{\lambda \mu}\left(\varepsilon^{\prime}\right) \sum_{S \sigma} 4 \pi i^{S} Y_{S \sigma}^{*}\left(\varepsilon_{\mathrm{ph}}\right) \sum_{l m} Y_{l m}^{*}(\varepsilon) \ldots \\
& \ldots \int d \Omega Y_{\lambda \mu}^{*}(\theta \varphi) Y_{S \sigma}(\theta \varphi) Y_{l m}(\theta \varphi) \int d r r^{2} \psi_{v \lambda}(r) j_{S}\left(2 k_{\mathrm{ph}} r\right) \psi_{n l}(r) \\
& =\sum_{\lambda \mu} \sum_{S \sigma} \sum_{l m} I_{\mathrm{ang}}^{(f-f)} I_{\mathrm{rad}}^{(f-f)} \\
& I_{\mathrm{ang}}^{(\text {free-free })}=4 \pi i^{S} Y_{\lambda \mu}\left(\varepsilon^{\prime}\right) Y_{S \sigma}^{*}\left(\varepsilon_{\mathrm{ph}}\right) Y_{l m}^{*}(\varepsilon) \int d \Omega Y_{\lambda \mu}^{*}(\theta \varphi) Y_{S \sigma}(\theta \varphi) Y_{l m}(\theta \varphi) \\
& I_{\mathrm{rad}}^{(\mathrm{free}-\text { free })}=\int d r r^{2} \psi_{\nu \lambda}(r) j_{S}\left(2 k_{\mathrm{ph}} r\right) f_{n l}(r) \\
& =C\left(k^{\prime}, \lambda\right) C(k, l)\left(2 i k^{\prime}\right)^{\lambda}(2 i k)^{l} \int d r r^{2+l+\lambda+S} \exp \left[-i\left(k+k^{\prime}\right) r\right] j_{S}\left(2 k_{\mathrm{ph}} r\right) \ldots \\
& { }_{{ }_{1}} F_{1}\left(1+\lambda+i / k^{\prime}, 2+2 \lambda, 2 i k^{\prime} r\right){ }_{1} F_{1}(1+l+i k, 2+2 l, 2 i k r)
\end{aligned}
$$

Here again we must develop the spherical Bessel function using either an infinite power series (eq. 1.5b) or a finite sum of exponentials, (eq. 1.5c). In either case the integral is of the type in (eq. 3.7).

We have used both approaches, as a means of internal checking.

[1] A. R. B. de Castro, T. Laarmann, J. Schulz, H. Wabnitz, and T. Möller, Phys. Rev. A 72, 023410 (2005).

[2] T. Laarmann, A. R. B. de Castro, P. Gürtler, W. Laasch, J. Schulz, H. Wabnitz, and T. Möller, Phys. Rev. A 72, 023409 (2005).

[3] A. R. B. de Castro, T. Möller, H. Wabnitz, and T. Laarmann, Braz. J. Phys., 35, 632 (2005).

[4] A. R. B. de Castro, T. Laarmann, J. Schulz, H. Wabnitz, and T. Möller, Phys. Rev. A 74, 027402 (2006).

[5] T. Laarmann, A. R. B. de Castro, P. Gürtler, W. Laasch, J. Schulz, H. Wabnitz, and T. Möller, Phys. Rev. A 74, 037402 (2006).

[6] A. Maquet, B. Piraux, A. Scrinzi, and R. Taïeb, Phys. Rev. A 74, 027401 (2006).

[7] D. Charalambidis, P. Tzallas, N. A. Papadogiannis, L. A. A. Nikolopoulos, E. P. Benis and G. D. Tsakiris, Phys. Rev. A 74, 037401 (2006).

[8] L. A. A. Nikolopoulos and P. Lambropoulos, Phys. Rev. A 74,
063410 (2006).

[9] J. J. Sakurai, "Modern Quantum Mechanics", Addison Wesley Publ Co, N. York (1994).

[10] E. Hairer and G. Wanner, "Solving ordinary differential equations II - stiff and differential/algebraic problems", Springer Verlag Heidelberg (1996).

[11] M. Nurhuda and F. H. M. Faisal, Phys. Rev. A 60, 3125 (1999).

[12] J. Bauer, L. Plucinski, B. Pireaux, R. Potvliege, M. Gajda, and J. Krzywinski, Journal of Physics B: At Mol. and Op. Phys. 34, 2245 (2001).

[13] L. Landau and E. Lifchitz, "Mécanique Quantique, Théorie non-relativiste", Ed Mir, Moscou 1966.

[14] A. R. Edmonds, "Angular Momentum in Quantum Mechanics", Princeton University Press, Princeton 1957.

[15] G. Arfken, "Mathematical Methods for Physicists", Academic Press, New York 1970. 